\title{
On the Geometry of Relativistic Anyon
}

\author{
A.Nersessianfl \\ Bogoliubov Laboratory of Theoretical Physics, JINR, \\ Dubna, Moscow district, 141980 Russia
}

PACS: 03.30.+p, 03.65.-w

\begin{abstract}
A twistor model is proposed for the free relativistic anyon. The Hamiltonian reduction of this model by the action of the spin generator leads to the minimal covariant model; whereas that by the action of spin and mass generators, to the anyon model with free phase space that is a cotangent bundle of the Lobachevsky plane with twisted symplectic structure. Quantum mechanics of that model is described by irreducible representations of the $(2+1)$-dimensional Poincare' group.
\end{abstract}

\footnotetext{
${ }^{*}$ Work is supported by INTAS-RFBR grant No. 95-0829.

†e-mail:nerses@thsun1.jinr.dubna.su
} 
1. Introduction. As is known, in $(2+1)$-dimensional space-time there can exist anyons, particles with arbitrary spins and statistics [四]. Quantum mechanics of anyons as a spinning particles is described by unitary irreducible representations of the $(2+1)$ dimensional Poincare' group [2]. However, classical theory of anyons cannot be described within the standard approach based on the introducing of Grassmann variables. For this purpose are use the so-called "minimal" and "extended" approaches (here me employ the classification of Ref.[3]).

In the minimal approach, the spin is provided by the nontrivial symplectic structure of phase space [4, 2]. The minimal relativistic-covariant model (in what follows, $K$-model) of the anyon with spin $s$ is realized on the phase space $T_{*} \mathbb{R}^{1.2}$ with the twisted symplectic structure

$$
g_{a b} d P^{a} \wedge d Q^{b}+s \frac{P^{a}}{P^{3}} \varepsilon_{a b c} d P^{b} \wedge d P^{c}, \quad P=\sqrt{P^{a} P_{a}}
$$

where $g_{a b}=\operatorname{diag}(1,-1,-1)$ is the metric of space $\mathbb{R}^{1.2}$ parametrized by the anyon relativistic momentum $P^{a}$; its position is defined by the fiber coordinates $Q^{a}$; the generators of Lorentz rotations $J^{a b}=\frac{1}{2} \varepsilon^{a b c} J_{c}$ are given by the relations

$$
J_{a}=\varepsilon_{a b c} P^{b} Q^{c}+s \frac{P^{a}}{P}
$$

and the phase space is restricted by the mass constraint: $P^{a} P_{a}=m^{2}$.

In the extended approach the spin is described by the extra phase degrees of freedom and it value is fixed by an appropriate constraints [3, 5, 7].

A shortcoming of minimal models is their being nonlinear; whereas that of extended models is the nontrivial structure of phase space and constraints. As a result, even the description of the interaction of an anyon with an external electromagnetic field in those approaches is not a simple problem [6, 7].

In this note, we propose a twstor model of a free relativistic anyon that has no drawbacks of both extended and minimal models. The phase space of this model (in what follows, $T$-model) is the twistor space; whereas the constrains fixing the anyon mass $m$ and spin $s$ and the $(2+1)$-dimensional Poincare' group define a linear symplectic transformations.

The Hamiltonian reduction of the $T$ - model by the action of the spin generator results in the $K$-model both at the classical and quantum level.

The Hamiltonian reduction of the $T$-model by the action of the spin and mass generators leads to the minimal model of an anyon with a free phase space. Its phase space is a cotangent bundle of the Poincare' (or Klein) model of the Lobachevsky plane $\mathcal{L}=S U(1.1) / U(1)$ with the twisted symplectic (pseudo-Kähler) structure and generators of the Poincare' group define its isometries $(\mathcal{L}$-model). The anyon quantum mechanics in the $\mathcal{L}$-model is described by the reducible representation of the $S U(1.1)$ group on the Lobachevsky plane playing the role of the momentum space. It can be obtained by the reduction of quantum-mechanical $T$-model.

2. $T$-model. Consider the twistor space $\mathbf{T}=T \mathbb{C}^{1.1}$ with the symplectic structure

$$
\Omega=i \eta_{\alpha \bar{\beta}}\left(d \pi^{\alpha} \wedge d \bar{\omega}^{\bar{\beta}}+d \omega^{\alpha} \wedge d \bar{\pi}^{\bar{\beta}}\right), \quad \alpha, \beta,=0,1
$$


where $\eta$ - is the Pauli matrix $\sigma^{3}$ (or $\sigma^{2}$ ), playing the role of the metric on $\mathbb{C}^{1.1}$, the spinor indeces $\alpha, \beta$ are raised and lowered by use of this metric.

On this space, we define the linear symplectic action of the $(2+1)$-dimensional Poincare' group

$$
\left\{P^{a}, P^{b}\right\}=0, \quad\left\{P^{a}, J^{b}\right\}=\varepsilon^{a b c} P_{c},\left\{J^{a}, J^{b}\right\}=\varepsilon^{a b c} J_{c}
$$

where

$$
P^{a}=\pi^{\alpha} \sigma_{\alpha \bar{\beta}}^{a} \bar{\pi}^{\bar{\beta}} \quad J^{a}=\frac{1}{2} \sigma_{\alpha \bar{\beta}}^{a}\left(\pi^{\alpha} \bar{\omega}^{\bar{\beta}}+\omega^{\alpha} \bar{\pi}^{\bar{\beta}}\right),
$$

and vector indices $a, b, c=0,1,2$, (or $a, b, c=0,1,3$ at $\eta=\sigma^{2}$ ) are raised and lowered by use of the metric $g_{a b}=\frac{1}{2} \operatorname{tr}\left(\eta \sigma^{a} \eta \sigma^{b}\right)$, where $\sigma_{\alpha \bar{\beta}}^{0}=\delta_{\alpha \bar{\beta}}$.

It can be verified easily that the invariants of the Poincare'group obey the equalities $P^{a} P_{a}=P^{2}, \quad P_{a} J^{a}=P J$, where

$$
\begin{gathered}
P=\pi^{\alpha} \eta_{\alpha \bar{\beta}} \bar{\pi}^{\bar{\beta}}, \quad J=\frac{1}{2} \eta_{\alpha \bar{\beta}}\left(\pi^{\alpha} \bar{\omega}^{\bar{\beta}}+\omega^{\alpha} \bar{\pi}^{\bar{\beta}}\right), \\
\{P, J\}=0, \quad\left\{P, P^{a}\right\}=\left\{P, J^{a}\right\}=0, \quad\left\{J, P^{a}\right\}=\left\{J, J^{a}\right\}=0 .
\end{gathered}
$$

Therefore functions $J$ and $P$ can be interpreted as generators of the anyon spin and mass, whereas the constraints $P^{a} P_{a}=m^{2}(>0), \quad P^{a} J_{a}=m s$ fixing the anyon mass $m$ and spin $s(c=\hbar=1)$ can be replaced by the equivalent ones

$$
J=s, P=m, \text { or } J=-s, P=-m,
$$

which generate a linear transformations of the twistor space.

Note that the constraints reduce the number of (phase) degrees of freedom down to four, and consequently, completely define the dynamics of free anyon. The first pair of equations (8) changes into the second pair via the discrete canonical transformation corresponding to spatial reflections. Thus we restrict ourselves by the consideration of the first pair of constraints in (8), putting $m>0$.

We will call below the above-described model of anyon on the twistor space the $T$-model.

Let us perform the Hamiltonian reduction of the $T$-model by the action of the spin generator $J$.

Since the generators

$$
X^{a}=\frac{i}{2} \sigma_{\alpha \bar{\beta}}^{a}\left(\pi^{\alpha} \bar{\omega}^{\bar{\beta}}-\omega^{\alpha} \bar{\pi}^{\bar{\beta}}\right)
$$

obey the condition $\left\{X^{a}, J\right\}=0$, we take coordinates of the reduced (six-dimensional) space to be the functions $P^{a}, Q^{a}=\frac{X^{a}}{2 P}$.

As a result, upon reduction of the Poisson brackets and generators $J^{a}$ we obtain the $K$-model described in the Introduction.

Now consider the quantum-mechanical $T$-model of the anyon in the momentum representation

$$
\bar{\omega}_{\alpha}=-\frac{\partial}{\partial \pi^{\alpha}}, \quad \omega^{\alpha}=\frac{\partial}{\partial \bar{\pi}_{\alpha}}
$$

and impose, on the wave function $\Psi(\pi, \bar{\pi})$, the analogs of constraints (8):

$$
P \Psi(\pi, \bar{\pi})=m \Psi(\pi, \bar{\pi}), \quad J \Psi(\pi, \bar{\pi})=s \Psi(\pi, \bar{\pi}) .
$$


For reduction to the $K$-model we solve the second equation of (11) by the substitution

$$
\Psi_{s}(p, \bar{p})=\psi_{s}\left(P^{a}\right) \mathrm{e}^{i s \gamma}, \quad[J, \gamma]=i .
$$

Thus $\psi_{s}\left(P^{a}\right)$ represents the wave function of the anyon in the $K$-model, and the coordinates $Q^{a}$ are quantized as follows:

$$
\hat{Q}^{a}=-i \frac{\partial}{\partial P^{a}}-s A_{a}(P), \quad A_{a}(P)=\left\{Q_{a}, \gamma\right\} .
$$

Choosing, for example, $\gamma=\log \pi^{0} / \bar{\pi}^{0}$, we shall see, that $A_{a}(P)$ is similar to the potential of Dirac monopole, and has the unique singularity in $P^{0}=P$ point.

Note that the generators $P^{i}=\left(P, P^{a}\right), J^{i j}=-J^{j i}=\left(J^{0 a}=X^{a}, J^{a b}=\frac{1}{2} \epsilon^{a b c} J_{c}\right)$ satisfy the relations $P^{i} P_{i}=0,\left\{J, P^{i}\right\}=\left\{J, M^{i j}\right\}=0$ and define the algebra

$$
\left\{P^{i}, P^{j}\right\}=0, \quad\left\{P^{k}, J^{i j}\right\}=2 g^{k[i} P^{j]}, \quad\left\{J^{i j}, J^{k l}\right\}=2 g^{k[i} M^{j] l}-2 g^{i[k} M^{l] i},
$$

where $g_{i j}=\operatorname{diag}\left(g_{a b},-1\right)$. .

Thus at $\eta=E, a=1,2,3$ they give the well-known twistor representation of the $(3+1)$-dimensional Poincare' algebra for massless particles, and $J$ represents the helicity operator. This explains the repeatedly mentioned resemblance between the anyon covariant model and the free model of a 3-dimensional massless particle.

3. $\mathcal{L}$-model. Solution of the mass constraint in the $K$-model produces a noncovariant model of the anyon with the cotangent bundle of the Lobachevsky plane as a free (without constraints) phase space. However, it is more convenient to derive it directly from the $T$-model through its Hamiltonian reduction by the action of generators $P$ and $J$ with values (8). Owing to the commutativity of these generators, the reduced space is fourdimensional, and consequently, it is a physical phase space of the anyon.

The complex coordinates of the reduced space $\left(p, z^{*}\right)$ can be take to be the functions

$$
p=\pi^{1} / \pi^{0}, \quad z^{*}=\left\{p, \omega^{\alpha} \bar{\omega}_{\alpha}\right\}=\lambda^{1}-\omega \lambda^{0}, \quad \lambda^{\alpha}=\omega^{\alpha} / \pi^{0},
$$

so that the reduced space turns out to be the tangent bundle $T \mathcal{L}$ of the Lobachevsky plane $\mathcal{L}=S U(1.1) / U(1)$ in the Poincare' model (or in the Klein model at $\eta=\sigma^{2}$ ) (see Appendix). We identify $T \mathbb{C}^{1.1}$ with cotangent bundle $T_{*} \mathcal{L}$ by passing to the coordinates $p, z=i g(p, \bar{p}) \bar{z}^{*}$, where $g(p, \bar{p}) d p d \bar{p}$ is the metric of the Lobachevsky plane.

In these coordinates the symplectic structure complying with the reduced Poisson brackets is of the form

$$
\Omega^{\mathrm{red}}=d z \wedge d p+d \bar{z} \wedge d \bar{p}+i \frac{s}{m} g(z, \bar{z}) d z \wedge d \bar{z}, .
$$

The generators $P^{a}$ are reduces to the generators of the $S U(1.1)$ action on $\mathcal{L}$ (see Appendix A), and $J^{a}$ are reduced to the form

$$
J^{a}=V^{a}(p) z+\bar{V}^{a}(\bar{p}) \bar{z}+\frac{s}{m} P_{a},
$$

where $V^{a}(p)=i \partial_{p} P^{a}(p, \bar{p})$ define the isometries of the Käller structure on $\mathcal{L}$. 
So, the physical phase space of a free massive relativistic anyon is the cotangent bundle of the Lobachevsky plane with a twisted symplectic structure.

Quantum $T$-model (10) can be reduced to the quantum $\mathcal{L}$-model, e.g. by the substitution

$$
\Psi(\pi, \bar{\pi})=\psi_{m, s}(p, \bar{p}) e^{i s \gamma},
$$

which immediately results in

$$
\hat{z}=i \frac{\partial}{\partial p}+\frac{s}{m} \frac{\partial K(p, \bar{p})}{\partial p},
$$

where $K(p, \bar{p})$ is the Kähler potential of the Lobachevsky plane.

THe role of wave function in such a quantum $\mathcal{L}$-model plays $\psi(p, \bar{p})$, and the generators $J^{a}$ define the (reducible) representation of the $(2+1)$-dimensional Lorentz group on the Lobachevsky plane.

Note is to be made that this representation has arisen in the extended models of anyons from quantization of the isotopic space, where the parameter $p$ played the role of isotopic coordinate.

4.Conclusion. So, we have presented the twistor model for a free relativistic anyon and have established correspondence between that model and the minimal covariant model of anyon. We have found that the physical phase space of a classical free anyon is a cotangent bundle of the Lobachevsky plane with a twisted symplectic (pseudo-Kähler) structure (the $\mathcal{L}$-model), and its quantum mechanics is described by irreducible representations of the $(2+1)$-dimensional Poincare' group.

Just as a $(3+1)$-dimensional massless particle is dual to the system "charge-Dirac monopole", a massive relativistic anyon is dual to a nonrelativistic particle moving on the Lobachevsky plane in a presence of constant uniform magnetic field.

The $T$-model can be easily generalized for the anyon on the (anti-)de-Sitter space. For this purpose note, that the generators $J^{a}, P_{ \pm}^{a}=\sigma_{\alpha \bar{\beta}}^{a}\left(\pi^{\alpha} \bar{\pi}^{\bar{\beta}} \pm \omega^{\alpha} \bar{\omega}^{\bar{\beta}}\right)$, realize the algebras so(2.2) and so(1.3) on the twistor space $\mathbf{T}$. In analogy with the $T$-model, one can replace the invariants $P_{ \pm}^{a} P_{a \pm}-J^{a} J^{a}, P_{ \pm}^{a} J_{a}$ by $J, P_{ \pm}=\pi^{\alpha} \bar{\pi}_{\alpha} \pm \omega^{\alpha} \bar{\omega}_{\alpha}$. The phase space of the analog of the $K$-model is again $T_{*} \mathbb{R}^{1.2}$ with symplectic structure (1). However, the momentum and mass generators are determined in analogy with the Runge-Lenz vector and the Hamiltonian in the "charge -dyon" (MIC-Kepler) system [8], but not by the $P^{a}, P$ generators; the physical phase space is $\mathcal{L} \times \mathcal{L}$.

Note that in the framework of the $T$-model, on can easily to formulate the problem of interaction of a relativistic anyon with an external uniform electromagnetic field, which became a subject of discussion [7], but was not solved compleetely even at the classical level.

Acknowledgements. The author is grateful to A.Karabegov, S.Lyakhovich and A.Polychronakos for useful discussions as well as to E.Ivanov, A.Pashnev, V.Ter-Antonyan, and O.Khudaverdian for interest to the work. 


\section{Appendix. Lobachevsky plane as a reduced space.}

Consider the space $\mathbb{C}^{1.1}$ with the pseudo-Kähler 2-form $\Omega^{0}=i d \pi^{\alpha} \wedge d \bar{\pi}_{\alpha}$, where $\bar{\pi}_{\alpha}=$ $\eta_{\alpha \beta} \bar{\pi}^{\bar{\beta}}$, and the metric $\eta$ of this space is the Pauli matrice.

The symplectic action of group $U(1.1)$ on this space is given by the Hamiltonians

$$
P=\pi^{\alpha} \bar{\pi}_{\alpha}, \quad P^{a}=\pi^{\alpha}\left(T^{a}\right)^{\beta} \alpha \bar{\pi}_{\beta}: \quad \Omega_{0}^{-1}\left(d P, d P^{a}\right)=0, \quad \Omega_{0}^{-1}\left(d P^{a}, d P^{b}\right)=\varepsilon^{a b c} P_{c},
$$

where $T^{a}=\sigma^{a} \eta, \sigma^{a}=\left(\sigma^{0}, \ldots \neq \eta, \ldots 3\right)$, and indices are raised and lowered by use of the metric $g_{a b}=\operatorname{tr} T^{a} T^{b}=\operatorname{diag}(+1,-1,-1)$.

The Hamiltonian reduction of $\mathbb{C}^{1.1}$ by the action of $U(1)$ given by the Hamiltonian $P$ corresponds to the factorization of the level surface $P=m$ by the $U(1)$ group action. Thus the reduced space is two-dimensional, and the (complex) functions

$$
p=\frac{\pi^{1}}{\pi^{0}} \quad, \bar{p}^{+}=\frac{\bar{\pi}_{1}}{\bar{\pi}_{0}}
$$

can play the role of its local coordinates (when $m \neq 0$ ). The action of the group $S U(1.1)$ on the reduced space is given by linear-fractional transformations.

The reduced Poisson bracket is determined by the relations

$$
\left\{p, \bar{p}^{+}\right\}_{0}^{\text {red }}=\left.\left\{p(\pi), \bar{p}^{+}(\bar{\pi})\right\}_{0}\right|_{P=m}=-\frac{i}{m}\left(1+p \bar{p}^{+}\right)^{2}
$$

so that

$$
g(p, \bar{p}) d p d \bar{p}^{+}=m /\left(1+p \bar{p}^{+}\right)^{2} d p d \bar{p}^{+}
$$

defines the Kähler structure with the potential $K=m \log \left(1+p p^{+}\right)$on the reduced space.

The symplectic action of the group $S U(1.1)$ on the reduced space is generated by generators in $P^{a}$, restricted to the level surface $P=m$.

Let $\eta=\sigma^{3}$. Then $\bar{p}^{+}=-\bar{p}$, and $|p|<1(|p|>1)$ for $m>0(m<0)$, i.e. the reduced space is the Poincare'model of the Lobachevsky plane.

The generators $P^{a}$ on this space are of the form

$$
P^{0}=m \frac{1+p \bar{p}}{1-p \bar{p}}, \quad P^{1}=m \frac{p+\bar{p}}{1-p \bar{p}}, \quad P^{2}=m \frac{i(p-\bar{p})}{1-p \bar{p}},
$$

and generates the isometries of Kähler structure $\Omega_{0}^{-1}\left(d P^{a}, \quad\right)=V^{a}(p) \partial_{p}+\bar{V}^{a}(\bar{p}) \partial_{\bar{p}}$

Let $\eta=\sigma^{1}$, then $\bar{p}^{+}=-\frac{1}{\bar{p}}$, and for $m>0(m<0)$ we have $(\operatorname{Im} p>0)(\operatorname{Im} p<0)$ : the reduced space is the Klein model of the Lobachevsky plane.

Analogously, at $\eta=\sigma^{1}$ we obtain the Klein model described by the right (left) halfplane of the complex plane.

Note that the mapping of the interior of the circle into its exterior in the Poincare'model (the upper half-plane into the lower one in the Klein model), $m \rightarrow-m, \omega \rightarrow \frac{1}{\omega}$, is corresponds to the spatial reflections: $P^{0} \rightarrow P^{0}, P^{1} \rightarrow P^{1}, P^{2} \rightarrow-P^{2}$. 


\section{References}

[1] J.F. Schonfeld, Nucl.Phys. B185, 117(1981);

F.Wilczek, Phys.Rev.Lett. 48, 1144 (1982); ibid. 49,957 (1982)

[2] R.Jakiw, V.P. Nair, Phys.Rev. D43, 1933 (1991)

[3] J.L.Cortes, M.S.Plyushchay, Int.J.Mod.Phys. A11, 3331 (1996)

[4] B.-S. Skagerstam, A. Stern, Int.J.Mod.Phys.A5, 1575 (1990)

[5] M.S. Plyushchay, Phys.Lett.B248, 107 (1990); ibid.B320, 91 (1994)

I. Gorbunov, S. Kuzenko, S. Lyakhovich, hep-th/9607114

[6] C.Chou, V.P.Nair, A.P. Polychronakos, Phys.Lett. B304, 105(1993)

[7] M. Chaichian, R. Gonzalez Felipe, D. Luis-Martinez, Phys.Rev.Lett. 71, 3405 (1993); ibid. 74, 2009(1994)

R.Jakiw, V.P. Nair, ibid. 73, 2007(1994)

[8] A.Nersessian, V.Ter-Antonyan, Mod.Phys.Lett.A9, 2431(1994) 